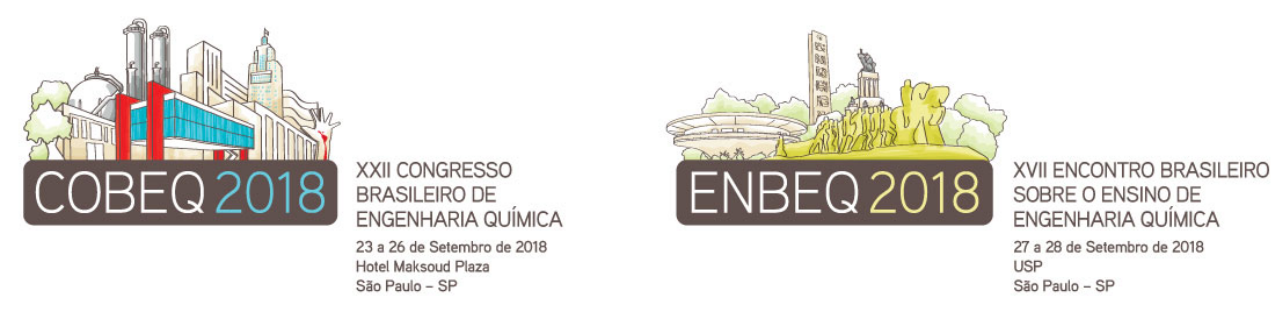

\title{
AVALIAÇÃO DA AÇÃO ANTI-INCRUSTANTE DE SURFACTANTES INCORPORADOS EM UMA MATRIZ PARA TRATAMENTO DA BIOINCRUSTAÇÃO (BIOFOULING) EM AMBIENTES MARINHOS
}

\author{
MEDEIROS AO ${ }^{1}$, SILVA MGC ${ }^{2,3}$, ALMEIDA DG ${ }^{1,3}$, MEIRA HM${ }^{1,3}$ e SARUBBO LA ${ }^{1,2,3}$ \\ ${ }^{1}$ Universidade Católica de Pernambuco, Centro de Ciência e Tecnologia - UNICAP/CCT \\ ${ }^{2}$ Universidade Federal Rural de Pernambuco, Rede Nordeste de Biotecnologia - RENORBIO; \\ ${ }^{3}$ Instituto Avançado de Tecnologia e Inovação - IATI \\ E-mail para contato: anderson_medeiros.oli@hotmail.com
}

\begin{abstract}
RESUMO - Em ambientes marinhos costeiros, a comunidade biológica presente se desenvolve nas superfícies imersas de diferentes estruturas, causando prejuízos econômicos às instalações locais. Para remoção desses organismos, são utilizados biocidas tóxicos que causam efeitos deletérios em seres vivos não-alvo. Desse modo, este trabalho objetivou formular um anti-incrustante não biocida contendo biossurfactantes obtidos de processo fermentativo e pela modificação química do óleo de soja (Glycine max (L.)), e avaliar seu comportamento antifouling frente a organismos incrustantes marinhos. Para este fim, foi preparada uma matriz à base de resina natural contendo os biossurfactantes, a qual foi aplicada em placas metálicas para testes de imersão no Porto do Recife-PE durante 25 dias. Os resultados analisados da área incrustada através do software ImageJ demonstraram que a matriz + laurato e matriz + ácido hidroxilado, ainda que amena, exibiram as atividades antifouling mais promissoras.
\end{abstract}

\section{INTRODUÇÃO}

A bioincrustação superficial de materiais imersos no ambiente marinho é um problema que afeta particularmente as indústrias e equipamentos do setor marítimo (Gittens et al., 2013; Gule; et al., 2016). A comunidade biológica marinha incrustante (biofouling), desenvolve-se nesses substratos artificiais submersos, em quatro fases: geração de uma camada de moléculas orgânicas; colonização primária por biofilmes (vírus, bactérias, fungos e diatomáceas); colonização unicelular por esporos de algas; e fixação de macroincrustantes multicelulares (Gule; et al., 2016).

Revestimentos anti-incrustantes à base de produtos químicos sintéticos é a estratégia primária para combater o biofouling na indústria marinha. Biocidas contendo o TBT (tributilestanho) apesar de eficazes na redução da incrustação, estão vetados por serem prejudiciais para outros organismos do ambiente marinho além dos organismos incrustantes (Gittens et al., 2013). Em termelétricas próximas ao mar, por exemplo, é imprescindível a aplicação de biocidas (como o hipoclorito de sódio - $\mathrm{NaOCl}$ ) na água de resfriamento que circula nos equipamentos para controlar o biofouling. 


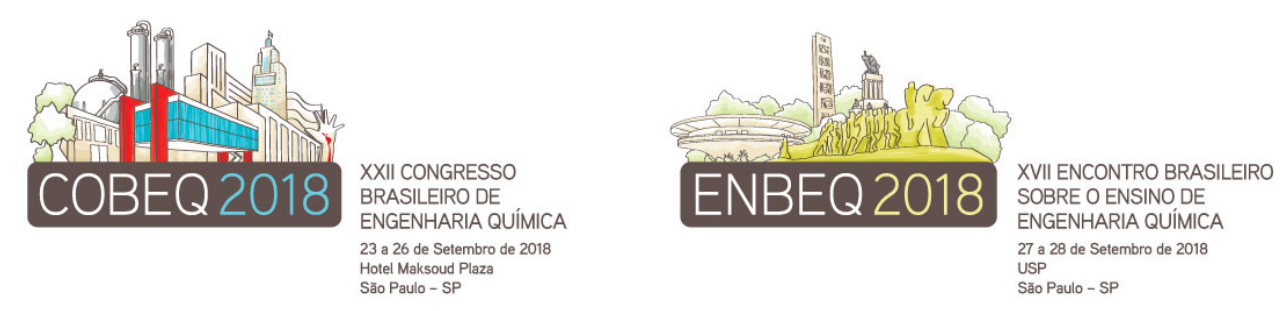

Para evitar ou reduzir as bioincrustações em superfícies imersas, uma das tendências atuais é a alteração de superfícies para torná-las mais hidrofóbicas por meio do uso de substâncias tensoativas, como também pela adição de substâncias naturais com ação antimicrobiana nas superfícies tratadas, eliminando, assim, a adesão microbiana, pois uma vez que biofilmes colonizam uma superfície, eles alteram as propriedades da superfície e facilitam o processo de adesão de outros incrustantes (Gittens et al., 2013; Damodaran e Murthy, 2016).

Os surfactantes são compostos químicos que possuem propriedades anfipáticas. Tal característica os permite interagir com distintas moléculas de diferentes afinidades e com isso serem utilizados em diversas aplicações. Podem ser obtidos por derivados do petróleo (maioria dos surfactantes disponíveis no mercado), por vegetais e por micro-organismos. Dentre as várias vantagens dos biossurfactantes sobre os surfactantes sintéticos estão a baixa toxicidade, biodegradabilidade, atividade e resistência em condições extremas de temperaturas, $\mathrm{pH}$ e salinidade. Além de sua atividade antimicrobiana, esses compostos também provaram serem bons inibidores da adesão microbiana e formação de biofilmes (Santos et al., 2016; Araujo et al., 2016).

Posto isto, o presente trabalho teve por objetivo formular e avaliar anti-incrustante contendo surfactantes naturais atóxicos com potencial anti-biofouling para aplicação como revestimento em qualquer instalação submersa em água do mar com presença de organismos incrustantes.

\section{MATERIAIS E METODOS}

\subsection{Obtenção dos ativos}

As substâncias avaliadas no presente trabalho foram selecionadas com base em estudos que demonstraram o potencial antimicrobiano/anti-incrustante de alguns tipos de surfactantes (Fusetani, 2011; Rufino et al., 2011). Foram produzidas: o ácido oleico hidroxilado, sal de sódio do ácido oleico hidroxilado, oleato de glicerila, laurato de glicerila e oleato hidroxilado de glicerila, por meio da modificação química do óleo de soja (Glycine max (L.)) através de reações de neutralização, esterificação do glicerol e reações de hidroxilação por epoxidação, utilizando catalisador adequado. O ramnolipídeo foi obtido a partir da bactéria Pseudomonas cepacia (Soares da Silva et al.,2017) e o surfactante/emulsificante lecitina adquirido da empresa Nutryervas do Brasil LTDA.

\subsection{Formulação da matriz}

A composição da formulação da matriz foi adaptada de Acevedo et al. (2013) e expressa em porcentagem por massa. Os componentes foram misturados em um agitador mecânico (Tecnal LTDA, Brasil) a $2000 \mathrm{rpm}$ por $50 \mathrm{~min}$. Os compostos ativos testados foram incorporados posteriormente à matriz na concentração de $10 \%(\mathrm{p} / \mathrm{p})$.

\subsection{Ensaios de imersão}

Ensaios de imersão in situ foram realizados no Porto do Recife S.A., Recife/PE. Neste local, placas de metal $(1 \mathrm{~mm} \times 100 \mathrm{~mm} \times 200 \mathrm{~mm})$ contendo as matrizes experimentais foram imersas em uma área com alta ocorrência de organismos incrustantes. As placas foram verificadas inicialmente após 7 dias e depois em 25 dias de imersão. As imagens obtidas das placas passaram por análise da superfície com a ajuda do software editor de imagem "ImageJ". 

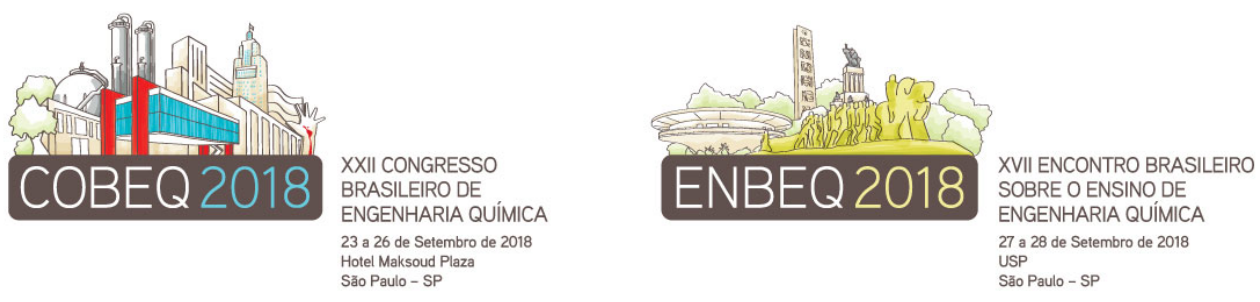

\section{RESULTADOS E DISCUSSÕES}

Na Figura 1 (Figuras 1A - 1B), estão apresentados os resultados obtidos nos testes em campo, após 7 dias (A) e 25 dias (B) das placas imersas no Porto do Recife. O retardo da incrustação foi evidenciado pela diferença na cobertura das placas, conforme mostrado na Figura 1.

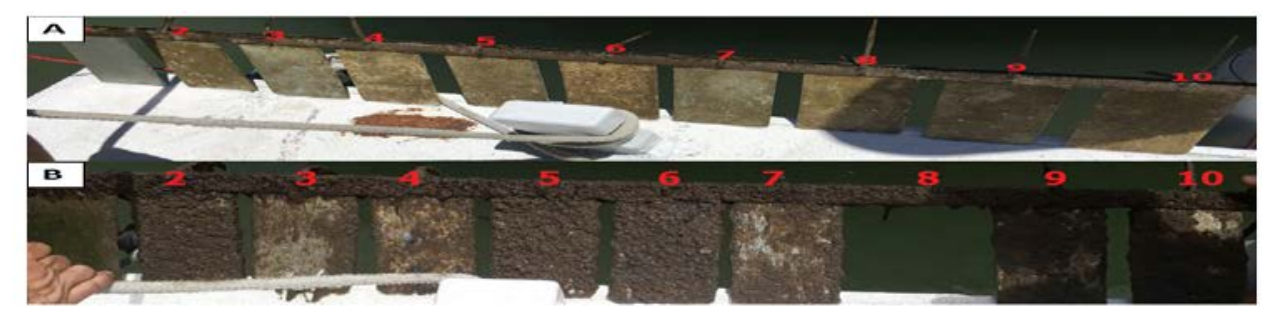

Figura 1 - Placas após 7 dias (A) e 25 dias (B) imersas no Porto do Recife. 1: matriz, 2: surfactante oligômero do glicerol, 3: laurato de glicerila, 4: ácido oleico hidroxilado, 5:ramnolipídeo, 6:matriz teste sem carbonato de cálcio/óxido de zinco,7: lecitina, 8: sal de sódio do ácido oleico hidroxilado, 9: oleato hidroxilado de glicerila e 10: sem revestimento/tratamento.

Na Figura 2 é apresentada a análise dos percentuais de área coberta das placas, calculados pelo software Image após 25 dias. A aferição pelo aplicativo indicou que, em comparação com as placas 1 (95,75\%) e 2 (91,04\%), completamente cobertas, as placas $3(56,81 \%)$ e $4(72,34 \%)$ apresentaram retardo da incrustação.
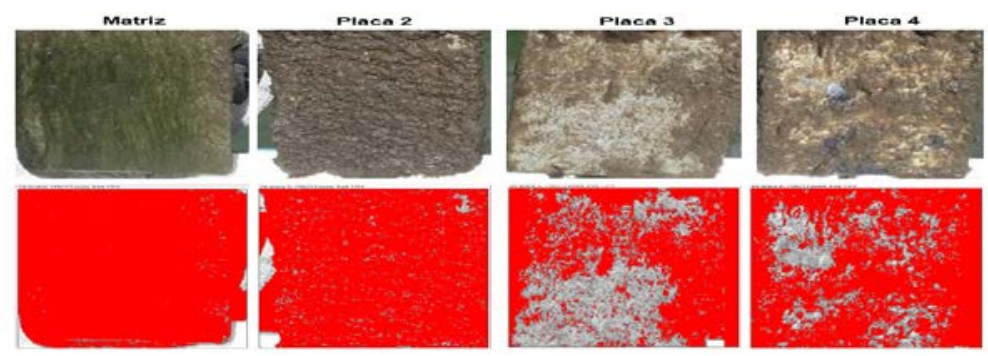

Figura 2 - Análise de área da superfície coberta pelo software ImageJ das placas após 25 dias submergidas no Porto do Recife, Recife - PE. Imagens superiores: placas reais. Imagens inferiores: análise estatística de área coberta das placas correspondentes.

Para evitar o biofouling, duas abordagens podem ser empregadas: (I) evitar o desenvolvimento das fases iniciais da bioincrustação empregando bioativos em forma não letal para o meio ambiente, e (II) formular e aplicar revestimentos anti-incrustantes inibidores da fixação larval, tornando a superfície não adequada para o desenvolvimento do biofouling (Bressy et al., 2014; Jerabek et al., 2016). Neste trabalho, as duas estratégias foram simultaneamente aplicadas, e como resultado, o surfactante laurato de glicerila (placas 3) e ácido oleico hidroxilado (placas 4) demonstraram potencial de inibição, modesto, do desenvolvimento do biofouling. Acevedo et al. (2013) também avaliaram uma matriz natural, adicionada, todavia, de extratos de animais marinhos (esponja e pepino-do-mar) durante 90 dias de imersão, obtendo atividade anti-biofouling. No presente estudo, no entanto, foram obtidos surfactantes bioativos de fontes renováveis (óleo de soja e processo fermentativo), os quais podem ser produzidos industrialmente em grande escala, evitando, assim, utilizar animais marinhos que necessitariam de criação em cativeiro, processo lento e pouco produtivo. 

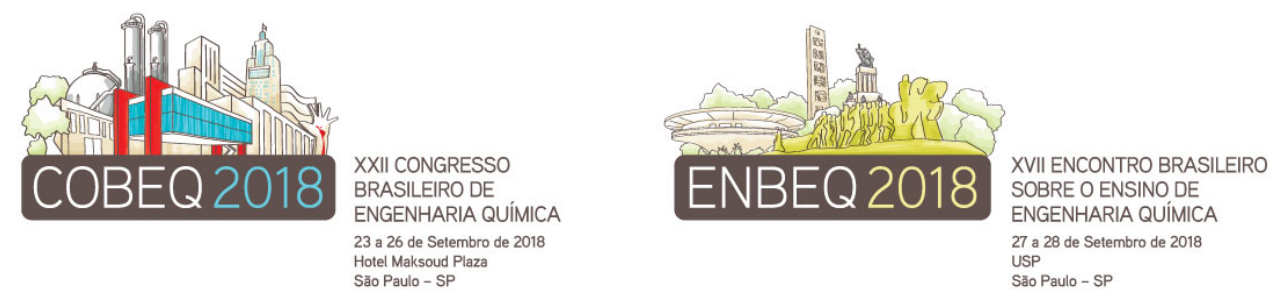

\section{CONCLUSÃO}

As composições testadas da Matriz, Matriz + laurato e Matriz + ácido hidroxilado apresentaram atividade antifouling discreta/comedida/modesta/razoável? durante o período testado. Os resultados também demonstraram que é possível obter surfactantes naturais com atividade antifouling a partir de fontes naturais, sem a necessidade de extrair diretamente de organismos marinhos, evitando assim, a degradação da fauna e flora marinhas.

\section{REFERÊNCIAS}

ACEVEDO, M. S. et al. Antifouling paints based on marine natural products from Colombian Caribbean. Inter. Biodet. \& Biodeg., v. 83, p. 97-104, 2013.

ARAUJO, L. V. DE et al. Rhamnolipid and surfactin: Anti-adhesion/antibiofilm and antimicrobial effects. F. Con., v. 63, p. 171-178, maio 2016.

BRESSY, CHRISTINE; LEJARS, M. Marine Fouling: An Overview Marine Fouling An Overview. T. Jour. of Oc. Tec. v. 9, n. February, 2014.

DAMODARAN, V. B.; MURTHY, N. S. Bio-inspired strategies for designing antifouling biomaterials. Bios Res. v. 20, n. 1, p. 18, dez. 2016.

FUSETANI, N. Antifouling marine natural products. Nat. Prod. Rep., v. 28, n. 2, p. 400-410, 2011.

GITTENS, J. E. et al. Current and emerging environmentally-friendly systems for fouling control in the marine environment. Biot. Adv. v. 31, n. 8, p. 1738-1753, 2013.

GULE, N. P.; BEGUM, N. M.; KLUMPERMAN, B. Advances in biofouling mitigation: A review. C. R. E. S. and T. v. 46, n. 6, p. 535-555, mar. 2016.

JERABEK, A. S.; WALL, K. R.; STALLINGS, C. D. A practical application of reduced-copper antifouling paint in marine biological research. Peer J, v. 4, p. e2213, 2016.

RUFINO, R. D. et al. Antimicrobial and anti-adhesive potential of a biosurfactant Rufisan produced by Candida lipolytica UCP 0988. Coll. and Surf. B: Biointerf., v. 84, n. 1, p. 1-5, 2011.

SANTOS, D. et al. Biosurfactants: Multifunctional Biomolecules of the 21st Century. Inter. Jour. of Mol. Sci. v. 17, n. 3, p. 401, 18 mar. 2016.

SOARES DA SILVA, R. DE C. F. et al. Production and characterization of a new biosurfactant from Pseudomonas cepacia grown in low-cost fermentative medium and its application in the oil industry. Bio. and Agri. Biotec. v. 12, n. September, p. 206-215, 2017. 